\title{
A complication of diabetes: easily confused, easily missed
}

\author{
Authors: Gopal Chattopadhyay, ${ }^{A}$ Anupam Maity, ${ }^{B}$ Pratik Bhowmik, ${ }^{B}$ Sayan Das ${ }^{B}$ and Kalyan Kumar Gangopadhyay ${ }^{B}$
}

\section{Introduction}

A 60-year-old woman with long-standing type 2 diabetes mellitus (T2DM), hypertension and hypothyroidism presented with progressive pain and swelling of her left thigh for nearly 2 weeks without any fever. She had, without benefit, received courses of several antibiotics on the suspicion of cellulitis prior to presentation at our set-up. Capillary sugar at presentation was $>500 \mathrm{mg} / \mathrm{dL}$ ( $28 \mathrm{mmol} / \mathrm{L})$. Examination revealed diffuse swelling over the anterolateral aspect of the upper third of the left thigh, with erythema and tenderness without local hyperthermia. Her $\mathrm{HbA}_{1 \mathrm{c}}$ was $10.7 \%(93.4 \mathrm{mmol} / \mathrm{mol})$ and her estimated glomerular filtration rate was $30 \mathrm{~mL} / \mathrm{min} / 1.73 \mathrm{~m}^{2}$. Leukocytosis and elevated C-reactive protein prompted further use of antibiotics, again suspecting cellulitis or pyomyositis. However, improvement remained elusive. Poor response to antibiotics, and lack of evidence of pus necessitated further radiological evaluation. Magnetic resonance imaging (MRI) showed necrotic muscle tissue, suggesting a diagnosis of diabetic myonecrosis. She responded to supportive care and adequate control of blood sugar levels and was subsequently discharged. On initial follow-up, she was found to have fair glycaemic control without recurrence of her symptoms. However, she failed to maintain the follow-up beyond 6 months. We sadly learnt later that she had died about a year after her presentation to us.

\section{Discussion}

Diabetic myonecrosis or diabetic muscle infarction is a rare complication of long-standing and insufficiently controlled diabetes mellitus. ${ }^{1,2}$ It involves ischaemic necrosis of skeletal muscle, and commonly affects the proximal lower limb. A gamut of common differentials causes it to be frequently underdiagnosed. ${ }^{2,3}$

The affected region is painful and occasionally swollen. ${ }^{1-3}$ Lab parameters are not reliably consistent. ${ }^{2} \mathrm{MRI}$ is the investigation of choice. ${ }^{1-3}$ Patients generally respond over weeks to supportive therapy and adequate rest. ${ }^{1-3}$

The purpose of this report is to draw the attention of physicians to a rare complication of diabetes mellitus that mimics common diabetes-related conditions and pain syndromes. The crux appears to be a remarkable 5-year mortality, despite apparent complete resolution of the index episode. $^{2,3}$

\section{Conflicts of interest}

None declared.

\section{References}

1 Trujillo-Santos AJ. Diabetic muscle infarction: an underdiagnosed complication of long-standing diabetes. Diabetes Care 2003;26: 211-5.

2 Choudhury BK, Saikia UK, Sarma D, Saikia M et al. Diabetic myonecrosis: an underreported complication of diabetes mellitus. Indian J Endocrinol Metab 2011;15(Suppl 1):S58-61.

3 Cumberledge J, Kumar B, Rudy D. Risking life and limb: a case of spontaneous diabetic muscle infarction (diabetic myonecrosis). J Gen Intern Med 2016;31:696-8.

Authors: ${ }^{A}$ Epsom and St Helier University Hospitals NHS Trust, London, UK; ${ }^{\mathrm{P}}$ Peerless Hospitex Hospital and Research Center, Kolkata, India 